\title{
Review of a Series of Disc Lens Implants
}

\author{
J. M. KEENAN and J. L. PEARCE \\ Redditch
}

\begin{abstract}
Summary
A consecutive series of 380 patients (431 eyes) implanted with the Pearce Lasadisc Lens are reviewed, of which 225 patients ( 255 eyes) underwent detailed assessment. The operative technique for implanting the disc lens is described and the importance of superior capsular bag fixation is discussed. Postoperatively $89.9 \%$ of eyes achieved a best corrected visual acuity of $6 / 12$ or better. Five $(1.2 \%)$ of the implants were removed; three due to lens decentration causing visual problems, one after dislocating into the anterior chamber, and one after causing pupil block glaucoma. One implant dislocated into the vitreous.
\end{abstract}

The first intraocular lens, described and implanted by Ridley in 1949, was a biconvex circular lens of unpolymerized polymethylmethacrylate (PMMA), with a diameter of 8.3 millimeters and a central thickness of 2.4 millimeters. ${ }^{1}$ His concept was to place the lens in its most natural position behind the iris, relying for support on the intact posterior capsule behind and the iris diaphragm in front. ${ }^{2}$ Epstein modified this lens; the new lens was of larger diameter (9.4 millimeters) and thinner central thickness (1.5 millimeters). ${ }^{3}$ Ridley later changed to using anterior chamber lenses becuase of the high incidence of late posterior dislocation of his posterior chamber lenses. $^{2}$

The revival of interest in extracapsular cataract surgery was accompanied by an increase in interest in posterior chamber lenses. ${ }^{4}$ There were many available lens designs including posterior chamber lenses based on Binkhorst's and Federov's prepupillary implants with the optic placed in the posterior chamber, and rigid and flexible loop posterior chamber lenses. ${ }^{5}$
Galand described a variation in the method of extracapsular cataract extraction, which he termed the envelope technique. ${ }^{6}$ He noted the main complication with different styles of posterior chamber implant was decentration of the intraocular lens leaving the edge of the optic visible within the undilated pupil. The best centration was achieved by the almost circular Anis lens and the rigid Pearce tripod lens, raising the possibility of developing a circular rigid configuration as the basis for a stable implant. ${ }^{7}$

Galand et $a l .{ }^{8}$ designed and described a lenticular implant of PMMA with a biconvex, 7.0 millimeter optic and a total diameter of 9.0 millimeters which they termed a 'disc lens'. The theoretical advantages they proposed for this lens, which is implanted completely within the capsular bag, were that it would not dislocate out of the bag, would not act as a spring in the eye, would not cause pupillary or capsular bag capture, and would provide a 360-degree barrier effect against proliferating lens epithelium.

A rigid disc lens was also designed by

Department of Ophthalmology, The Alexandra Hospital, Woodrow South, Redditch B98 7UB.

Corrrespondence to: J. M. Keenan FCOphth, Birmingham and Midland Eye Hospital, Church Street, Birmingham B3 2NS. 
Pearce (Lasadisc Lens) and has been the subject of a preliminary report. ${ }^{9}$ This present paper details the clinical experience of a series of 380 consecutive patients (431 eyes) implanıed with the Lasadisc lens.

\section{Patients Materials and Methods}

The Lasadisc lens (Rayner) is a lathe cut, plano-convex lens, manufactured from ultraviolet absorbing PMMA. A 5.0 millimeter optical zone is surrounded by a 2.0 millimeter rim with a single dialling hole (Fig. 1). It may be inserted with either the convex surface (reverse optic mode) or the concave surface (laser-gap mode) posteriorly, theoretically lessening the risk of developing posterior capsule thickening or facilitating neodymium: YAG laser (YAG) capsulotomy respectively.

The Lasadisc lens was implanted in 380 patients (431 eyes) between 1986 and 1989 at the Alexandra Hospital, Redditch. There were 123 males and 257 females with an age range from nine to 98 years (mean 70 years). The disc lens was implanted bilaterally in 51 patients; 36 had bilateral surgery under the one anaesthetic, and 15 had their surgery performed with an intervening period between operations. Combined trabeculectomy, cataract extraction, and disc lens implant was undertaken in 14 eyes $(3.2 \%)$.

General anaesthesia was used for 323 procedures $(74.9 \%)$. Local anaesthesia using a peribulbar technique was used for the remainder; this group included 41 day cases. There were $208(48.3 \%)$ right eyes and $223(51.7 \%)$

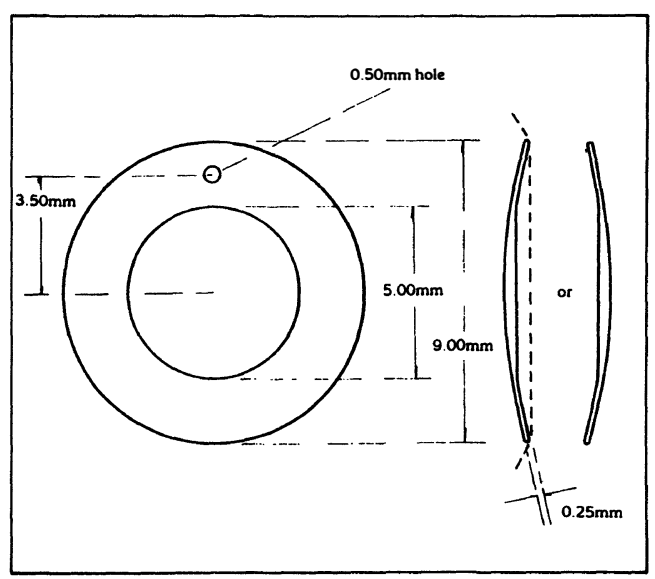

Fig. 1. Lasadisc lens. left eyes. Senile cataract was present in 403 eyes $(93.5 \%)$, posterior subcapsular lens opacities in $18(4.2 \%)$, congenital lamellar cataracts in nine $(2.1 \%)$, and there was one traumatic cataract. Biometry was performed on all eyes to compute the power of the intraocular lens.

Cyclopentolate $1 \%$, phenylephrine $10 \%$, and indomethacin $1 \%$ drops pre-operatively were used for dilation in the majority of eyes. One surgeon (JLP) performed $413(95.8 \%)$ of the implants; the remaining 18 were performed by surgeons of registrar grade.

At operation a superior rectus suture was placed. A fornix based conjunctival flap was formed and a posteriorly placed limbal incision was dissected forward into the anterior chamber. Sodium hyaluronate $1 \%$. was injected in the anterior chamber and a linear anterior capsulotomy from two o'clock to 10 o'clock was fashioned. The nucleus was hydrodissected with balanced salt solution and then manually expressed. A co-axial cannula was used for aspiration/irrigation and when necessary a modified cannula was used to remove residual cortical lens matter from the 12 o'clock position. The superior and inferior capsular bags were inflated with sodium hyaluronate.

The disc lens, with the dialling hole located superiorly and concave surface posteriorly, was inserted in the inferior capsular bag. A Sinskey hook was used to insert and exert posterior pressure on the superior portion of the disc lens using the dialling hole while the superior iris was simultaneously retracted with an iris retractor to view the superior capsular bag. After ensuring the disc lens was within the capsular bag superiorly a small inferior anterior capsulectomy was undertaken. The sodium hyaluronate was removed, and the wound closed with a continuous $9 / 0$ perlon suture with a buried central knot. The conjunctival flap was repositioned but not sutured. Subconjunctival antibiotics and steroids were administered in addition to topical timolol $0.5 \%$ drops and Isopto Carbachol 3\% drops. An eye pad and cartella shield were applied.

Dehisence of the posterior capsule, or rupture of the posterior capsule with vitreous loss, were considered contraindications to 
Table I Postoperative complications in total group of 431 eyes receiving disc lens implants

Posterior capsule thickening

Partial decentration of disc

Full decentration of disc

Dislocation of disc

Acute glaucoma

Superior pupil capture

Iris prolapse

Retinal detachment

Cystoid macular oedema

${ }^{*}$ Refers only to the group of 255 eyes reviewed.

disc lens implantation, and in the case of such an event an alternative lens style was implanted. A localised rupture of the posterior capsule without vitreous loss was not considered a contraindication to disc lens implantation.

All patients were examined on the first and third postoperative day and thereafter as indicated by clinical judgement. Topical steroid and antibiotic drops were used for four weeks postoperatively and then tailed off. Postoperative dilating drops were not routinely used. Refraction was performed and spectacles prescribed between six and ten weeks.

For the purpose of this study, all patients were recalled. 225 patients (255 eyes) responded and attended for examination which included visual acuity, applanation tonometry, slit lamp examination before and after pupil dilation, indirect ophthalmoscopy to detail the extent of the peripheral fundal view, and gonioscopy to determine the position of the implant in relation to the capsular bag. The initial patients attending for assessment had their eyes dilated with cyclopentolate $1 \%$ and phenylephrine $10 \%$ drops; this regime was later changed to tropicamide $1 \%$ and phenylephrine $2.5 \%$ drops, with thymoxamine $0.5 \%$ drops instilled after examination.

The position of the disc lens was assessed by examining the position of the lens optic against the red fundus reflex with the pupil dilated. This was assessed most easily by an indirect ophthalmoscope not utilising a lens; further information was sometimes obtained by utilising an indirect lens and by slit lamp examination. The lens position was described in four categories;

(1) central, when the lens optic was in a completely central position;

(2) partial decentration, where there was some decentration with the visual axis remaining within the optical zone of the lens;

(3) full decentration, when the lens was decentered to the extent that the visual axis was outside the optical zone; and

(4) dislocation, when the lens was dislocated into the vitreous or anterior chamber.

\section{Results}

Results were divided into two sections; the first reporting on the whole group (431 eyes) and the second, describing the group studied in more detail (255 eyes). The average length of follow up is sixteen months (range 2-38 months).

The operative complications consisted of a surgical hyphaema in four patients and a small posterior capsule rupture in two patients without vitreous loss. The surgical hyphaemas resolved spontaneously without complication.

The postoperative complications are detailed in Table I. Posterior capsule thickening was described when the degree of posterior capsule opacification was considered sufficient to interfere with vision. YAG laser capsulotomy was performed in 127 of these eyes at completion of the study, and the remaining 29 eyes were awaiting this procedure.

It was not possible to determine the number of eyes with partially decentered implants in the group (176 eyes) who did not attend for review. One of the cases of full decentration occurred in a patient who sustained a posterior capsule rupture at the time of surgery. A posterior capsule dehiscence is present in

Table II Postoperative visual acuities in 426 eyes receiving disc lens implants

\begin{tabular}{|c|c|c|c|c|c|c|c|c|}
\hline Visual acuity & $6 / 5$ & $6 / 6$ & $6 / 9$ & $6 / 12$ & $6 / 18$ & $6 / 24$ & $6 / 36$ & $6 / 60$ or less \\
\hline Number of cases & 57 & 83 & 127 & 61 & 36 & 13 & 10 & 39 \\
\hline$\%$ of total & $13.4 \%$ & $19.5 \%$ & $29.8 \%$ & $14.3 \%$ & $8.5 \%$ & $3.1 \%$ & $2.3 \%$ & $9.2 \%$ \\
\hline
\end{tabular}


Table III Causes of reduced visual acuity in the 98 eyes not achieving a corrected visual acuity of 6/12 or better

Age related macular degeneration 30

Posterior capsule thickening

Diabetic maculopathy

End stage open angle glaucoma

Amblyopia

Chronic uveitis with cystoid macualr oedema

Myopic degeneration

Corneal opacity

Vitreous haemorrhage

Endothelial dystrophy

Retinitis pigmentosa

Postoperative cystoid macular oedema

Postoperative retinal detachment

Traction retinal detachment*

Previous retinal detachment

Unexplained

*Secondary to diabetic retinopathy.

the patient with dislocation of the implant into the vitreous.

One case of acute glaucoma was due to a pupil block mechanism with the disc lens apposed to the posterior iris surface; the second was due to iris bombe secondary to $360^{\circ}$ posterior synechiae to the rim of remaining anterior capsule. Both were treated successfully, the former with YAG iridotomies and the latter with a surgical peripheral iridectomy.

Four patients with a history of chronic uveitis, now quiescent, one with a history of intermittent acute anterior uveitis associated with ankylosing spondylitis, and five patients with open angle glaucoma controlled on treatment, were implanted with the disc lens with no deterioration in their clinical course.

The last recorded visual acuities are detailed in Table II. The visual acuity was corrected with refraction or pin-hole for the 255 eyes examined in detail, the last recorded vision was used for the remaining 176 eyes. Five patients are excluded; three with decentered lenses which were replaced, one with lens dislocation into the vitreous, and one patient where there was no record of the postoperative visual acuity. The causes of reduced visual acuity in the 98 eyes not achieving 6/12 or better are outlined in Table III. The two cases with corneal endothelial dystrophy had their endothelial changes present pre-operatively. The ten patients in whom there was no obvious reason for reduced visual acuity belonged to the group whose notes only were reviewed.

A best corrected visual acuity of $6 / 12$ or better was achieved in $89.9 \%$ of eyes when preexisting ocular pathology as a cause of decreased postoperative acuity is excluded (Table IV). The causes of the decreased visual acuity in the remaining $10.1 \%$ (37 eyes) are shown in Table V.

The results of the 255 eyes (225 patients) attending recall and examined in more detail are charted in Table VI. The seven eyes with disc lens/iris apposition comprised three eyes with the implant apposed to the superior iris; three eyes with the implant apposed to the inferior iris; and one eye with the implant apposed to both the superior and inferior iris.

Following pupil dilation a view of the far peripheral retina was available in $239(94.5 \%)$ eyes. There was little image distortion caused by the joining of the disc lens optic and the supporting annulus. A restricted fundal view was present in 15 eyes; this was due to posterior synechiae of the iris to the anterior capsule, anterior lens surface, or both.

There were two complications which were related to dilating the pupils for examination. One patient (age 32) reported eye ache and decreased vision on the morning following pupil dilation. Examination revealed the disc lens freely mobile in the anterior chamber with the iris constricted behind it. The second patient (age 79) presented four days following pupil dilation with acute glaucoma secondary to pupil block, the lens being directly apposed to the posterior iris. Both lenses were removed and replaced with flexible loop posterior chamber lenses.

\section{Discussion}

A series of 380 patients (431 eyes) implanted

Table IV Best corrected postoperative visual acuities in 365 eyes receiving disc lens implants

\begin{tabular}{|c|c|c|c|c|c|}
\hline Visual acuity & $6 / 5$ & $6 / 6$ & $6 / 9$ & $6 / 12$ & $6 / 18$ or less \\
\hline Number of cases & 57 & 83 & 127 & 61 & 37 \\
\hline$\%$ of total & $15.6 \%$ & $22.7 \%$ & $34.8 \%$ & $16.7 \%$ & $10.1 \%$ \\
\hline
\end{tabular}


Table $\mathbf{V}$ Causes of postoperative visual acuity of 6/18 or less in eyes with no recognised preoperative pathology

Posterior capsule thickening

25

Postoperative cystoid macular oedema

Postoperative retinal detachment

Unexplained

1

10

with the Lasadisc lens are reviewed. They have been implanted in patients with a wide age range, with different types of cataract, and under general and local anaesthesia. Topical indomethacin was used pre-operatively and is useful in maintaining a large pupil during surgery which facilitates implantation of the disc lens. ${ }^{10}$

A corrected visual acuity of $6 / 12$ or better was achieved by $89.9 \%$ of eyes. This figure should be higher after YAG laser capsulotomy is performed on the 25 eyes with posterior capsule thickening. It might also be improved if the ten patients in whom there was no obvious cause recorded in the notes for a postoperative visual acuity of less than $6 / 12$ could be reviewed.

The operative complications detailed do not include cases with intent to implant a disc lens in which a change of lens type was made due to the intraoperative complications of capsule dehiscence or posterior capsule rupture with vitreous loss which occurred during nucleus expression, aspiration/irrigation of the soft lens matter, or attempted implantation of the disc lens. The number of these cases is not known due to the retrospective nature of this study in which only cases which had a disc lens implanted were reviewed.

The majority of the complications occurred early in the series of disc lens implants. It

Table VI Results of detailed examination of 255 eyes receiving disc lens implants

\begin{tabular}{lr}
\hline Postoperative superior capsular bag lens fixation: \\
Fixated & $147(57.6 \%)$ \\
Not fixated & $101(39.6 \%)$ \\
Not visualised & $7(2.7 \%)$ \\
Lens centration: & \\
Centered & $192(75.3 \%)$ \\
Partial decentration & $61(23.9 \%)$ \\
Disclocated & $1(0.4 \%)$ \\
YAG capsulotomies & \\
(including waiting list) & $115(45.1 \%)$ \\
Lens/iris apposition & $7(2.8 \%)$ \\
\hline
\end{tabular}

became obvious to us and others that the amount of anterior capsule that should be removed was critical, and that it was always necessary to ensure satisfactory superior capsular bag fixation. ${ }^{11}$ It became our policy to record in the operation notes that each disc lens implant was superior capsular bag fixated. 114 eyes were recorded as having the disc lens implant superior capsular bag fixated; 75 of these eyes were reviewed postoperatively (Table VII). This revealed that 61 $(81.3 \%)$ of the lenses remained superior capsular bag fixated. The remaining 39 eyes in this group included one eye from which the disc lens was removed due to full decentration; there were no other centration problems recorded.

The implant was superior capsular bag fixated on postoperative examination in 147 $(57.6 \%)$ of the 255 eyes reviewed in detail; this group had a significantly higher incidence of centration $(p=0.01)$ than the 101 eyes not superior capsular bag fixated postoperatively (Table VIII).

The majority $(81.3 \%)$ of the disc lenses which were superior capsular bag fixated at the time of surgery remained so postoperatively; and postoperative superior capsular bag fixation is associated with a high percentage $(95.9 \%)$ of central lenses. Secure superior capsular bag fixation must therefore be the aim when implanting the disc lens.

The three patients with full decentration of the disc lens suffered severe visual distortion requiring removal of the implant. The lenses were replaced with flexible loop posterior chamber lenses, and all achieved a corrected visual acuity of $6 / 9$. The patient with lens dislocation into the vitreous is successfully visually rehabilitated with a daily wear gas permeable contact lens.

The phenomenon of 'parachute lights' was experienced by 22 patients with partially decentered implants. This is a vivid descrip-

Table VII Postoperative superior capsular bag fixation in a group of 75 eyes with recorded operative superior capsular bag fixation of the disc lens implant

\begin{tabular}{lccc}
\hline Total & SCB & Not SCB & No View \\
\hline 75 & 61 & -11 & 3 \\
$(100 \%)$ & $(81.3 \%)$ & $(14.7 \%)$ & $(4.0 \%)$ \\
\hline
\end{tabular}

SCB $=$ Superior capsular bag fixated. 
Table VIII Relationship of postoperativesuperior capsular bag fixation to lens centration in 255 eyes with disclens implants

\begin{tabular}{lcccc}
\hline & Central & Partial Decentration & Dislocation & Row Total \\
\hline SCB & 141 & 6 & 0 & $147(57.6 \%)$ \\
Not SCB & 46 & 54 & 1 & $101(39.6 \%)$ \\
No view & 6 & 1 & 0 & $7(2.7 \%)$ \\
Column Total & 193 & 61 & 1 & $255(100 \%)$ \\
& $(75.7 \%)$ & $(23.9 \%)$ & $(0.4 \%)$ & \\
\hline
\end{tabular}

SCB $=$ Superior capsular bag fixated.

tion and remarkably similar from case to case. An alternate phrase alikened the phenomenon to the Prince of Wales feathers. It consists of a parachute shaped reflection above a light source with the canopy of the parachute consisting of a band of shimmering lights. It occurred in the more severe cases of partial decentration of the implant and was compatible with good visual acuity. None of the centered implants showed this phenomenon.

YAG capsulotomy was either performed on or was awaited in a total of 156 eyes $(36.2 \%)$. The majority of the disc lenses were implanted with the concave surface posteriorly (laser gap mode). A higher rate of posterior capsule opacification with disc lenses has previously been noted. ${ }^{11}$ This is considered to be due to the lack of posterior pressure by the implant on the posterior capsule. It may be possible to reduce the incidence of posterior capsule opacification by implanting the disc lens convex posteriorly (reverse optic mode).

The disc lens was in apposition to the posterior iris in seven eyes. None of these lenses was superior capsular bag fixated and only two were in the inferior capsular bag. The lenses apposed to the superior iris have a risk of pupil capture following dilation. The eyes with lenses not superior or inferior capsular bag fixated more seriously are at risk of pupil block glaucoma. One of these patients presented in the week following pupil dilatation with this condition. The remaining four eyes with disc lenses not superior or inferior capsular bag fixated remain at risk and should be considered for prohylactic YAG iridotomies.

A degree of caution should be used in dilating eyes with disc lens implants. The patient who returned with the disc lens dislocated into the anterior chamber was young and had been dilated with cyclopentolate $1 \%$ and phenylephrine $10 \%$ drops. The wide and lengthy pupil dilation must have favoured dislocation of the disc lens. The lens at the time of examination was noted to be central with superior and inferior capsular bag fixation, and had been in place for 24 months. The second patient, who presented with pupil block glaucoma secondary to disc lens apposition to the posterior iris, had been dilated with the modified regime of tropicamide $1 \%$ and phenylephrine $2.5 \%$ drops, with thymoxamine $0.5 \%$ drops instilled after examination. At examination the disc lens was neither superior or inferior capsular bag fixated and presumably pupil dilation was sufficient to allow a change in the lens positon. No further problems were encountered using the modified regime of pupil dilation. All patients were requested not to sit in a reading position or to lie prone until the effects of the dilating drops had worn off.

In summary, the Pearce Lasadisc lens has achieved good visual results in a large series of patients. Removal was required for five of the 431 disc lenses implanted, dislocation into the posterior segment occurred with one lens, and visual distortion described as parachute lights occurred in 21 patients with partially decentered lenses. These complications are directly related to the stability of the implanted disc lens, and they may be reduced with careful attention to superior and inferior capsular bag fixation.

We consider that the disc lens, possibly in the form of a modified design such as the compressible disc lens implant, ${ }^{12}$ does have a future. However, because of its potential for instability which requires both superior and inferior capsular bag fixation, it should be implanted only by those with experience in intercapsular cataract surgery. 
The authors are grateful to Mr D. E. Shaw, University Department of Ophthalmology, Birmingham, for statistical advice. They wish also to thank Mrs A. Badger, secretary to Mr J. L. Pearce, and Sister Jackson and the staff of the Out-patient Department, Alexandra Hospital, Redditch, for their helpful assistance.

Key words: Intercapsular cataract extraction; Intraocular disc lens; Intraocular lens centration; Posterior capsule opacification; Superior capsular bag fixation.

\section{References}

${ }^{1}$ Ridley H: Intra-ocular acrylic lenses. Trans Ophthalmol Soc UK 1951, 71: 617-21.

${ }^{2}$ Ridley H: Intra-ocular acrylic lenses-past, present and future. Trans Ophthalmol Soc UK 1964, 84: 5-14.

${ }^{3}$ Epstein E: Modified Ridley lenses. Br J Ophthalmol 1959, 43: 29-33.

${ }^{4}$ Pearce JL: Modern simple extracapsular surgery. Trans Ophthalmol Soc UK 1979, 99: 176-82.

${ }^{5}$ Pearce JL: Current state of posterior chamber intra- ocular lenses after intracapsular and extracapsular cataract surgery. Trans Ophthalmol Soc UK 1981, 101: 73-6.

${ }^{6}$ Galand A: A simple method of implantation within the capsular bag. Am Intra-ocular Implant Soc J 1983, 9: 330-2.

${ }^{7}$ Galand A: P J Hay Lecture: Present and future of implantation in the capsular bag. Eye 1988, 2: 336-40.

${ }^{8}$ Galand A and Delmelle M: Preliminary report on the rigid disc lens. J Cataract Refract Surg 1986, 12: 394-7.

${ }^{9}$ Watts MT and Pearce JL: Implantation of a disc lens in the capsular bag. Ophthalmol Surg 1988, 19: 546-48.

${ }^{10}$ Searle AET, Pearce JL, Shaw DE: Topical use of indomethacin on the day of cataract surgery. $\mathrm{Br} \mathrm{J}$ Ophthalmol 1990, 74: 19-21.

${ }^{11}$ Galand A, Feiss LJ, Delmelle M: The new disc lens in intercapsualr surgery. Implants in Ophthalmology 1988, 2: 234.

${ }^{12}$ Galand A: The Compressible Disc Lens Implant. Eur J Ref Surg 1990, 2: 82-5. 